\title{
Semantics of Concurrent Revisions
}

\author{
Sebastian Burckhardt and Daan Leijen \\ Microsoft Research
}

\begin{abstract}
Enabling applications to execute various tasks in parallel is difficult if those tasks exhibit read and write conflicts. We recently developed a programming model based on concurrent revisions that addresses this challenge in a novel way: each forked task gets a conceptual copy of all the shared state, and state changes are integrated only when tasks are joined, at which time write-write conflicts are deterministically resolved.

In this paper, we study the precise semantics of this model, in particular its guarantees for determinacy and consistency. First, we introduce a revision calculus that concisely captures the programming model. Despite allowing concurrent execution and locally nondeterministic scheduling, we prove that the calculus is confluent and guarantees determinacy. We show that the consistency guarantees of our calculus are a logical extension of snapshot isolation with support for conflict resolution and nesting. Moreover, we discuss how custom merge functions can provide stronger guarantees for particular data types that are tailored to the needs of the application.

Finally, we show we can visualize the nonlinear history of state in our computations using revision diagrams that clarify the synchronization between tasks and allow local reasoning about state updates.
\end{abstract}

\section{Introduction}

With the recent broad availability of shared-memory multiprocessors, many more application developers now have a strong motivation to tap into the potential performance benefits of parallel execution. Exploiting parallel hardware can be relatively easy if the application performs computations for which parallel algorithms are well known or straightforward to develop (such as for scientific problems or multimedia applications). However, traditional parallelization strategies often do not satisfactorily address how to execute different application tasks that access shared data in parallel.

For example, consider an office application that needs to perform five different tasks: (1) save a snapshot of the document to disk, (2) react to keyboard input by the user who is editing the document, (3) perform a spellcheck of the document, (4) render the document on the screen, and (5) exchange document updates with collaborating remote users.

Executing such tasks in parallel is not simple, because all of them potentially access the same data (such as the document) at the same time. For instance, in a case study on parallelizing a game application [3] we discovered that the parallel execution of the physics task and the render task is essential to achieve decent speedup on multiple cores. But these tasks naturally exhibit read-write conflicts: The physics task modifies 
all coordinates of game objects (to simulate elapsed time) while the render task reads all coordinates (to render a snapshot of the scene).

Avoiding, negotiating, or resolving such conflicts between parallel tasks can be quite challenging with traditional synchronization models. In fact, many programmers are deterred by the engineering complexity of performing explicit, manual synchronization (such as by using locks and critical sections) or replication (such as by creating temporary copies or using double buffering).

Our proposed programming model, concurrent revisions [8], simplifies parallelization of conflicting tasks by (conceptually) copying shared state automatically on a fork. Tasks execute in complete isolation because each has its own copy of the shared data (e.g. the document or the coordinates, in the above examples), somewhat analogous to source control systems that allow multiple programmers to work on the same code at the same time by creating local copies of files, and checking changed files back into the repository.

$$
\begin{aligned}
& x=0 ; \\
& y=0 ; \\
& r=\text { rfork }\{x=1 ;\} \\
& y=x ; \\
& \text { rjoin } r ;
\end{aligned}
$$

print $\mathrm{x}, \mathrm{y}$;

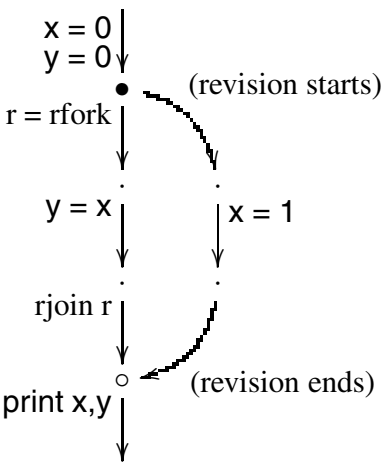

Fig. 1. An example of a revision diagram (on the right) representing the execution of a program (on the left). The effect of the write $x=1$ is confined to its revision until that revision is joined. Thus the print statement prints $(1,0)$.

For example, consider the code in Fig. 1 which illustrates the basic concept of forking and joining revisions and how to visualize executions using revision diagrams. The program on the left forks a concurrent revision, obtaining a handle $r$ which it later joins. The forked revision executes the assignment $x=1$, but the effect of this assignment is confined to that revision until it is joined, at which point all of its changes are applied to the joining revision. The diagram shows how the state is forked and joined (each vertex represents a state, and curved arrows represent fork and join), as well as how the state is locally updated by revisions (vertical arrows represent steps by revisions). Note that because revisions are isolated, data can flow only along edges in the diagram. Moreover, because the program specifies where to join revisions and does not depend on scheduling and timing, the execution is determinate.

Our previous work [8] has already provided some evidence that this concurrent revision model can be implemented efficiently enough to achieve satisfactory parallelization speedups, and that it is easier to use than locks or transactions [3]. However, our previous work left many important questions about the semantics unaddressed, in particular 
relating to determinacy and consistency guarantees. The purpose of our work presented in this paper is to address these questions rigorously and provide precise answers. We make the following contributions:

1. We give a minimal calculus describing the concurrent revision model. Because the calculus is small, it is well suited as a semantic reference and as an experimental tool to study various extensions or implementations. In fact, it was inspired (and is very similar to) the AME calculus [24] which served a similar purpose in the context of transactional memory. Note that we published a preliminary version of this calculus (without discussion or proofs) in the previous paper [8].

2. Even though the calculus is intrinsically concurrent, we prove that it guarantees determinacy.

3. We give a comprehensive discussion of consistency guarantees and state merging. We show that in the absence of write-conflicts and nesting, revisions are analogous to transactions with snapshot isolation. We also show how the introduction of custom merge functions into the calculus can allow the programmer to achieve stronger consistency guarantees tailored to the needs of the application.

4. We formalize the notion of a revision diagram (informally introduced in [8]). These diagrams capture the revision history of an execution, by showing the order and nesting of forks and joins. Moreover, they illustrate data flow, since information can propagate only along edges. We contrast revision diagrams with other commonly used task graphs (such as DAGs or SP-graphs) and prove that they are semilattices.

Overall, our work shows that the revision model preserves some of the best properties of sequential programs (deterministic execution, local reasoning about state updates) without forcing programmers to manually isolate parallel tasks, and without restricting parallel executions to be fully equivalent to a sequential execution. Rather, parallelism is expressed directly and explicitly, and always exploitable even if the tasks exhibit conflicts.

\section{Discussion}

We start with a high-level informal discussion of various aspects of the revision model, such as determinacy, nesting of revisions, handling of write-write conflicts, and revision diagrams. Moreover, we compare revisions to related work on transactional memory and determinacy.

\subsection{Revisions vs. Interleaved Tasks}

In our model, revisions are the basic unit of concurrency. They function much like asynchronous tasks that are forked and joined, and they may themselves fork and join other tasks. We chose the term 'revision' to emphasize the semantic similarity to branches in source control systems where programmers work with a local snapshot of the shared source code.

In particular, on every revisional fork (rfork), the system conceptually copies the entire state and each branch works on its own local copy. Every revision is completely 


\begin{tabular}{|c|c|c|}
\hline (sequential consistency) & (transactional memory) & (concurrent revisions) \\
\hline $\begin{array}{l}\mathrm{x}=0 ; \mathrm{y}=0 \\
\mathrm{t}=\text { fork }\{ \\
\quad \text { if }(\mathrm{x}=0) \mathrm{y}++\end{array}$ & $\begin{array}{l}\mathrm{x}=0 ; \mathrm{y}=0 \\
\mathrm{t}=\text { fork }\{\text { atomic }\{ \\
\quad \text { if }(\mathrm{x}=0) \mathrm{y}++\end{array}$ & $\begin{array}{l}x=0 ; y=0 \\
r=\text { rfork }\{ \\
\quad \text { if }(x=0) y++;\end{array}$ \\
\hline $\begin{array}{l}\} \\
\text { if }(y=0) x++ \\
\text { join } t\end{array}$ & $\begin{array}{l}\}\} \\
\text { atomic }\{\text { if }(y=0) x++;\} \\
\text { join } t\end{array}$ & $\begin{array}{l}\} \\
\text { if }(y=0) x++; \\
\text { rjoin } r\end{array}$ \\
\hline $\begin{array}{r}\operatorname{assert}((x=0 \wedge y=1) \vee \\
(x=1 \wedge y=0) \vee \\
(x=1 \wedge y=1))\end{array}$ & $\begin{array}{l}\text { assert }((x=0 \wedge y=1) \vee \\
\quad(x=1 \wedge y=0)) \\
;\end{array}$ & $\begin{array}{l}\operatorname{assert}(x=1 \wedge y=1) \text {; } \\
;\end{array}$ \\
\hline
\end{tabular}

Fig. 2. Outcomes under different programming models

isolated from the others and there is no possibility of communication through shared state. Any updates in a revision only become re-integrated once the revision is joined. Since there is no possibility of stateful interleavings with other threads, intra-revision reasoning (that is, reasoning about code executing within a revision) is sequential.

The revision model is a significant departure from memory models that interleave tasks at the level of individual instructions, such as sequential consistency [21]. Moreover, this difference is not simply a matter of the interleaving granularity. Transactional memory, for example, interleaves tasks at the granularity of atomic blocks [22, 15]. However, coarser interleaving does not in itself guarantee determinacy of executions, as the relative order of the atomic blocks is unspecified. Thus, whether we use sequential consistency or transactional memory, the interleaving chosen during an execution depends on nondeterministic arbitration which can vary between executions. In contrast, with our concurrent revision model, the precise structure of forks and joins is completely determined by the program and independent of runtime scheduling.

We illustrate this difference in Figure 2 where we compare the results of a program for these three models. The program forks a concurrent branch where each branch increments a variable $x$ or $y$ respectively depending on the value of the other variable ( $y$ and $x$ respectively). Under sequential consistency, there are many interleavings possible and there are three distinct possibilities for the values of $x$ and $y$. In the second program, we use transactional memory to limit the possible interleavings by executing each branch atomically. This effectively serializes the execution and we see either $x=0 \wedge y=1$ or $x=1 \wedge y=0$ depending on how the branches are scheduled. Using revisions, the outcome is always determinate: both branches get their own local (conceptual) copy of the state, and both branches will increment the variables ending in $x=1 \wedge y=1$.

\subsection{Local Reasoning vs. Serializability}

As Figure 2 shows, we can truly reason about each branch locally without considering any interleavings. However, note also that there is no equivalent sequential execution for this example. The lack of equivalence to some sequential execution is no accident: requiring such equivalence fundamentally limits the concurrency that can be practically 
exploited if tasks exhibit conflicts. For the kind of applications we have in mind, conflicts may be quite frequent.

With revisions, conflicts never destroy the available parallelism and never cause rollbacks. These choices provide substantial practical benefits over the use of rollbacks in optimistic transactional memory, which does not fare well in the presence of frequent conflicts, and cannot be easily combined with I/O [33].

Comfortably reasoning about application behavior in the absence of serializability requires understanding and conceptualizing a nonlinear history of state. We achieve this by introducing revision diagrams that directly visualize how the global state can be forked, updated, and joined (Fig. 1] Fig. 3). Revisions correspond to vertical chains in the diagram, and are connected by curved arrows that represent the forks and joins. We sometimes label the revisions with the actions they perform. Such diagrams visualize clearly how information may flow (it follows the edges) and how effects become visible upon the join. In Section 5 we show that the diagrams have a formal and well-defined meaning with relation to the calculus.

\subsection{State Merging}

When joining a revision, two copies of the state need to be merged together, which naturally raises two questions:

1. Can we always find a common ancestor state to help us determine if either side has made changes, and what those changes are?

2. If both sides have made changes, how do we resolve such write-write conflicts? (Note that there are no read-write or write-read conflicts between revisions.)

We answer the first question by showing how our calculus keeps track of the ancestor state (Section 3), and by showing that revision diagrams are semilattices and the ancestor state is in fact the greatest common ancestor (Section 5).

We address the second question by discussing several sensible merge policies. A key insight that makes state merging practical and convenient is that we need not define merge functions or policies globally, but can do so separately for each variable. In fact, we used this insight in previous work to parallelize a game application [8] by declaring the policy for each variable using special isolation types. Such isolation types allow the user to convey deep semantic knowledge that helps to exploit the available parallelism even if there are numerous conflicts.

In this paper, we consider a number of different merge policies. Note that these happen at the granularity of individual memory locations, not on the global state.

- (Join overwrites). This policy is the default in our basic calculus (Section 3). On a write-write conflict, the value of the joined revision overwrites the value of the joining revision.

- (Custom merge function). We can use a user-defined merge function to resolve conflicts deterministically (Section 4.1).

- (Give up and report). We can refuse to merge write-write conflicts and report the failure to the user, who can take some appropriate action. (Section 4.3). 
What we found a bit surprising is that the (Join overwrites)-policy is very useful in practice even though it appears to 'lose state'. This is because it lets us precisely control which revisions should take precedence over others by ordering the joins accordingly. For instance, if writes by revision B should take priority over writes by revision A, we can simply join B after joining A. The (Custom merge function)-policy was useful very specifically for implementing collections, which are often updated in a commutative way by concurrent revisions. We did not have any use for the (Give Up and Report)policy in the game.

Isolation types are also sensible from a software engineering perspective: in a large application an architect can annotate the shared data structures with their merge policy, while the code that uses such data types stays the same: in particular, programmers have no need to use atomic regions or locks when accessing such data types and can reason about it without considering interleaved executions.

\subsection{Nesting of Revisions}

Nesting of revisions is a natural consequence of the fact that revisions can themselves fork and join other revisions. We show a progression of nesting in the four left most examples of Fig. 3. The (regular) and (overlap) diagrams do not nest revisions beyond a depth of 1 (that is, only the main revision is forking and joining revisions). The (nested) diagram shows simple nesting, where a revision forks a child of depth 2 and then joins it (before being joined itself). The (bridge) diagram shows that child revisions can "survive" their parents (i.e. be joined later), and that revisions can be joined by a different revision than where they were forked.

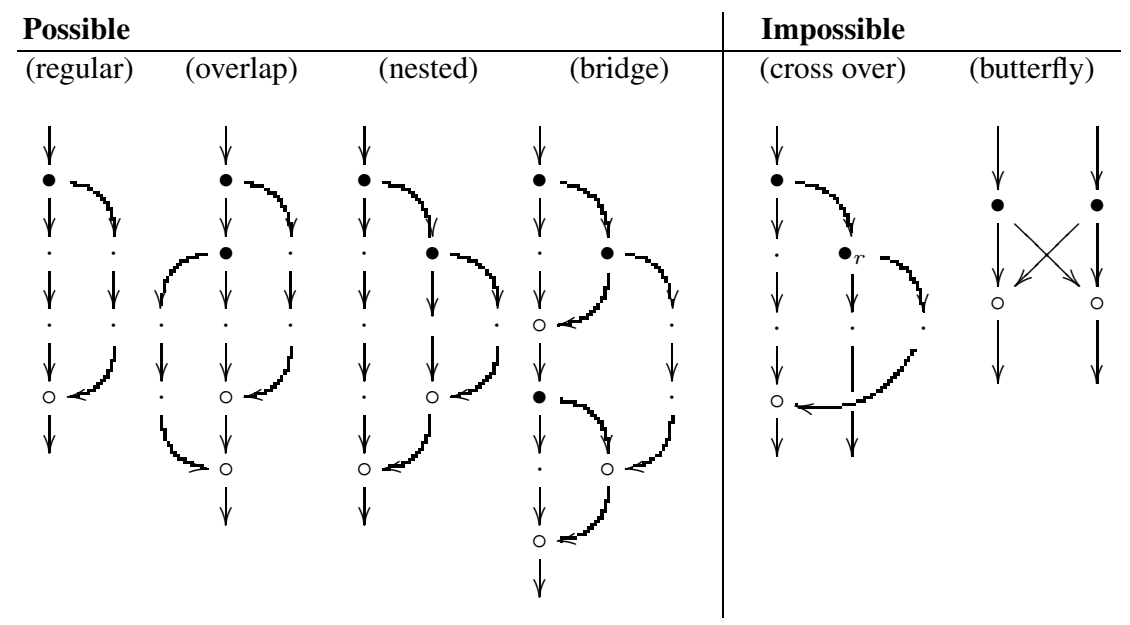

Fig. 3. Some examples of revision diagrams. The four on the left are all valid revision diagrams. On the right are two examples of impossible revision diagrams: the first one is not possible since the main branch cannot join on the outer revision as the (fresh) outer revision handle $r$ cannot be part of its state. The right-most diagram cannot be constructed for similar reasons, in particular, all revision diagrams are semi-lattices (Theorem 3). 
However, not all diagrams are possible, because revision handles must flow along edges. The two right-most examples in Fig. 3 show impossible revision diagrams. We prove some structural properties of revision diagrams in Section 5 in particular that revision diagrams are semi-lattices (Theorem 3 ).

Note that the structure of revision diagrams is entirely dynamic, not lexical. In particular, once a revision is forked, its handle can be stored in arbitrary data structures and be joined at an arbitrary later point of time. In some sense, revisions behave like futures whose side effects are delayed, and take effect atomically at the moment when the future is forced.

Although we present a fully dynamic model, it is of course possible to design a language that statically restricts the use of joins, to make stronger scheduling guarantees (as done in Cilk++ [14, 29]) or to simplify the most common usage patterns and to eliminate common user mistakes (as done in X10 [23]). In fact, many models (including an earlier version of our calculus) use a restricted "fork-join" parallelism [7,5]. Whether such restrictions are necessary or beneficial is beyond the scope of this paper. For now, we are content with stating that it is relatively easy to add them if desired, while it would be difficult to remove them from a calculus that depends on restrictive assumptions.

\subsection{Related Work}

Just as we do with revisions, proponents of transactions have long recognized that providing strong guarantees such as serializability [27] or linearizability [17] can be overly conservative for some applications, and have proposed alternate guarantees such as multi-version concurrency control [26] or snapshot isolation (SI) [4, 11, 30]. In fact, revisions can be understood as a natural generalization of snapshot isolation, extended to handle resolution of write-write conflicts following some policy (as discussed in Section 2.3), and to support nesting (as discussed in Section 2.4). We examine the relationship to snapshot isolation more formally in Section 4.3

There has been much prior work on programming models for concurrency [25, 12, 1 , 31, 2, 6]. Recently, many researchers have proposed programming models for deterministic concurrency [7, 5, 32, 28], creating renewed interest in an old problem previously known as determinacy [10]. All of these models differ semantically from revisions, and are quite a bit more restrictive. As they guarantee that the execution is equivalent to some sequential execution, they cannot easily resolve all conflicts on commit (like revisions do). Thus, they must restrict tasks from producing such conflicts either statically (by type system) or dynamically (pessimistic with blocking, or optimistic with abort and retry).

To the best of our knowledge, our combination of snapshot isolation and deterministic conflict resolution, as first presented in [8], is a novel way to simplify the parallelization of tasks that exhibit conflicts.

Isolation types are similar to Cilk++ hyperobjects [13]: both use type declarations by the programmer to change the semantics of shared variables. Cilk++ hyperobjects may split, hold, and reduce values. Although these primitives can (if properly used) achieve an effect similar to revisions, they do not provide a similarly seamless semantics. In particular, the determinacy guarantees are fragile, i.e. do not hold for all programs. For instance, the following program may finish with either $x==2$ or $x==1$ : 
reducer_opadd $\langle$ int $\rangle x=0$;

cilk_spawn $\{\mathrm{x}++\}$

if $(\mathrm{x}=0) \mathrm{x}++$;

cilk_sync

Isolation types are also similar to the idea of transactional boosting, coarse-grained transactions, and semantic commutativity [16, 19, 20], which eliminate false conflicts by raising the abstraction level. Isolation types go farther though: for example, the type versioned $\langle T\rangle$ does not just avoid false conflicts, but resolves true conflicts deterministically (in a not necessarily serializable way).

\section{Revision Calculus}

For reference and to remove potential ambiguities, we now present a formal calculus for revisions. It is based on a similar calculus introduced by prior work on AME (automatic mutual exclusion) [24].

Notations. To present the formal syntax and semantics succinctly, we use some standard and nonstandard notations for partial functions. For sets $A, B$, we write $A \rightarrow B$ for the set of partial functions from $A$ to $B$. For $f, g \in A \rightarrow B, a \in A, b \in B$, and $A^{\prime} \subset A$, we adopt the following notations: $f(a)=\perp$ means $a \notin \operatorname{dom}(f), \epsilon$ is the empty partial function with $\operatorname{dom}(\epsilon)=\varnothing, f[a \mapsto b]$ is the partial function that is equivalent to $f$ except that $f(a)=b$, and $f:: g$ is the partial function that is equivalent to $g$ on $\operatorname{dom}(g)$ and equivalent to $f$ on $A \backslash \operatorname{dom}(g)$. In our transition rules, we use patterns of the form $f\left(a_{1} \mapsto b_{1}\right) \ldots\left(a_{n} \mapsto b_{n}\right)$ (where $\left.n \geq 1\right)$ ) to match partial functions $f$ that satisfy $f\left(a_{i}\right)=b_{i}$ for all $1 \leq i \leq n$.

\subsection{Syntax and Semantics}

We show the syntax and semantics of our calculus concisely in Fig. 4 , The syntax (top left) represents a standard functional calculus, augmented with references. References can be created (ref $e$ ), read $(! e)$ and assigned $(e:=e)$. The result of a fork expression rfork $e$ is a revision identifier from the set Rid, and can be used in a rjoin $e$ expression (note that $e$ is an expression, not a constant, thus the revision being joined can vary dynamically).

To define evaluation order within an expression, we syntactically define execution contexts (Fig. 4 right column, in the middle). An execution context $\mathcal{E}$ is an expression "with a hole $\square$ ", and as usual we let $\mathcal{E}[e]$ be the expression obtained from $\mathcal{E}$ by replacing the hole $\square$ with $e$.

The operational semantics (Fig. 4 bottom) describes transitions of the form $s \rightarrow_{r} s^{\prime}$ which represent a step by revision $r$ from global state $s$ to global state $s^{\prime}$. Consider first the definition of global states (Fig. 4 top right). A global state is a partial function from revision identifiers to local states: there is no shared global state. The local state has three parts $(\sigma, \tau, e)$ : the snapshot $\sigma$ is a partial function that represents the initial state that this revision started in, the local store $\tau$ is a partial function that represents all the locations this revision has written to, and $e$ is the current expression. 


\section{Syntactic Symbols}

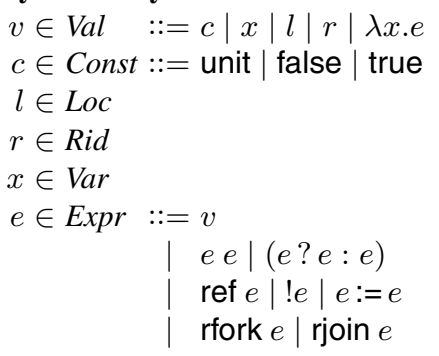

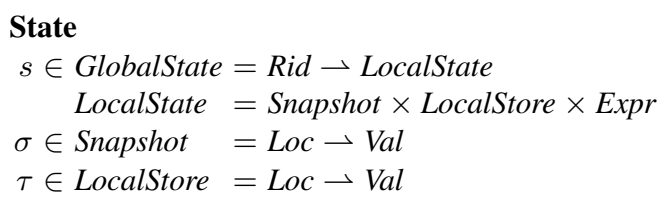

\section{Execution Contexts}

$\mathcal{E}=$

$\mathcal{E} e|v \mathcal{E}|(\mathcal{E} ? e: e)$

$\operatorname{ref} \mathcal{E}|! \mathcal{E}| \mathcal{E}:=e \mid l:=\mathcal{E}$

rjoin $\mathcal{E}$

\section{Operational Semantics \\ (apply) $s(r \mapsto\langle\sigma, \tau, \mathcal{E}[(\lambda x . e) v]\rangle) \quad \rightarrow_{r} s[r \mapsto\langle\sigma, \tau, \mathcal{E}[[v / x] e]\rangle]$ \\ (if-true) $s\left(r \mapsto\left\langle\sigma, \tau, \mathcal{E}\left[\left(\right.\right.\right.\right.$ true $\left.\left.\left.\left.? e_{1}: e_{2}\right)\right]\right\rangle\right) \rightarrow_{r} s\left[r \mapsto\left\langle\sigma, \tau, \mathcal{E}\left[e_{1}\right]\right\rangle\right]$ \\ (if-false) $s\left(r \mapsto\left\langle\sigma, \tau, \mathcal{E}\left[\left(\right.\right.\right.\right.$ false $\left.\left.\left.\left.? e_{1}: e_{2}\right)\right]\right\rangle\right) \rightarrow_{r} s\left[r \mapsto\left\langle\sigma, \tau, \mathcal{E}\left[e_{2}\right]\right\rangle\right]$

$\begin{array}{llll}(\text { new }) & s(r \mapsto\langle\sigma, \tau, \mathcal{E}[\text { ref } v]\rangle) & \rightarrow_{r} s[r \mapsto\langle\sigma, \tau[l \mapsto v], \mathcal{E}[l]\rangle] & \text { if } l \notin s \\ \text { (get) } & s(r \mapsto\langle\sigma, \tau, \mathcal{E}[! l]\rangle) & \rightarrow_{r} s[r \mapsto\langle\sigma, \tau, \mathcal{E}[(\sigma:: \tau)(l)]\rangle] & \text { if } l \in \operatorname{dom}(\sigma:: \tau) \\ (\text { set }) & s(r \mapsto\langle\sigma, \tau, \mathcal{E}[l:=v]\rangle) & \rightarrow_{r} s[r \mapsto\langle\sigma, \tau[l \mapsto v], \mathcal{E}[\text { unit }]\rangle] \text { if } l \in \operatorname{dom}(\sigma:: \tau) \\ & & \rightarrow_{r} s\left[r \mapsto\left\langle\sigma, \tau, \mathcal{E}\left[r^{\prime}\right]\right\rangle\right]\left[r^{\prime} \mapsto\langle\sigma:: \tau, \epsilon, e\rangle\right] \text { if } r^{\prime} \notin s \\ (\text { fork }) & s(r \mapsto\langle\sigma, \tau, \mathcal{E}[\text { rfork } e]\rangle) & \\ & & \\ \text { (join) } & s\left(r \mapsto\left\langle\sigma, \tau, \mathcal{E}\left[\text { rjoin } r^{\prime}\right]\right\rangle\right)\left(r^{\prime} \mapsto\left\langle\sigma^{\prime}, \tau^{\prime}, v\right\rangle\right) & \rightarrow_{r} s\left[r \mapsto\left\langle\sigma, \tau: \tau^{\prime}, \mathcal{E}[\text { unit }]\right\rangle\right]\left[r^{\prime} \mapsto \perp\right] \\ \text { (join }) & s\left(r \mapsto\left\langle\sigma, \tau, \mathcal{E}\left[\text { rjoin } r^{\prime}\right]\right\rangle\right)\left(r^{\prime} \mapsto \perp\right) & \rightarrow_{r} \epsilon\end{array}$

Fig. 4. Syntax and Semantics of the revision calculus

The rules for the operational semantics (Fig. 4, bottom) all follow the same general structure: a transition $s \rightarrow_{r} s^{\prime}$ matches the local state for $r$ on the left, and describes how the next step of revision $r$ changes the state.

The first three rules (apply), (if-true), and (if-false)) reflect standard semantics of application and conditional. They affect only the local expression. The next three rules (new), (get), and (set) reflect operations on the store. Thus, they affect both the local store and the local expression. The (new) rule chooses a fresh location (we simply write $l \notin s$ to express that $l$ does not appear in any snapshot or local store of $s$ ). The last two rules reflect synchronization operations. The rule (fork) starts a new revision, whose local state consists of (1) a snapshot that is initialized to the current state $\sigma:: \tau$, (2) a local store that is the empty partial function, and (3) an expression that is the expression supplied with the fork. Note that (fork) chooses a fresh revision identifier (we simply write $r \notin s$ to express that $r$ is not mapped by $s$, and does not appear in any snapshot or local store of $s$ ). The rule (join) updates the local store of the revision that performs the join by merging the snapshot, master, and revision states (in accordance with the declared isolation types), and removes the joined revision. We call $r$ the joining revision (or joiner), and $r^{\prime}$ the joined revision (or joinee). A join can only proceed if the joinee has executed all the way to a value (which is ignored). The final rule $\left(\right.$ join $\left._{\epsilon}\right)$ is added to prevent joining a revision handle more than once. If a revision handle is joined 
a second time, the joinee is no longer in the domain of $s$, and the entire state transitions to a special error state represented by the empty partial function $\epsilon$ (this state can not be reached in any other way, and has no outgoing transitions).

\subsection{Executions}

As usual, we let $\rightarrow$ be the union of all $\rightarrow_{r}$ where $r \in$ Rid. Furthermore, we use the following notations for repeated steps: we say $s \rightarrow^{n} s^{\prime}$ if $s^{\prime}$ can be reached from $s$ in exactly $n \rightarrow$-steps, we say $s \rightarrow^{*} s^{\prime}$ (transitive reflexive closure) if it can be reached in zero or more steps, $s \rightarrow^{+} s^{\prime}$ (transitive closure) if it can be reached in one or more steps, and $s \rightarrow ? s^{\prime}$ (reflexive closure) if it can be reached in zero or one steps.

We define global executions of expressions as follows. First, an expression $e$ is a program expression if it does not contain any revision identifiers (expressions may contain revision identifiers during execution, but not initially). We say a sequence of transitions $s_{0} \rightarrow s_{1} \rightarrow \cdots \rightarrow s_{n}$ is an execution of a program expression $e$ if $s_{0}=\{(r,(\epsilon, \epsilon, e)\}$ for some $r \in$ Rid. We call such an execution maximal if there exists no $s^{\prime}$ such that $s_{n} \rightarrow s^{\prime}$. Finally, given a program expression $e$ we write $e \downarrow s$ if there exists a maximal execution for $e$ with final state $s$.

\subsection{Determinacy}

A surprising property of our calculus is that executions are determinate and not dependent on a specific 'schedule'. Before we can state this precisely, we need a notion of equivalence of states modulo renaming of revisions and locations.

For a permutation $\alpha$ of Rid and a global state $s$ let $\alpha(s)$ be the global state obtained by replacing all revision identifiers $r$ that occur in $s$ with $\alpha(r)$. Similarly, define $\beta(s)$ for a permutation $\beta$ of Loc. We say two states $s, s^{\prime}$ are equivalent upto $\alpha \beta$-renaming, written as $s \approx s^{\prime}$, if there exist permutations $\alpha$ of Rid and $\beta$ of Loc such that $s=\alpha\left(\beta\left(s^{\prime}\right)\right)$.

We now state the main result of this section: executions are determinate modulo renaming of locations and revisions.

Theorem 1 (Determinacy). Let e be a program expression, and let $e \downarrow s$ and $e \downarrow s^{\prime}$. Then $s \approx s^{\prime}$.

Before proving this theorem, we make a few observations, and establish a few lemmas and an important confluence theorem.

Note that some executions may terminate in the special error state $\epsilon$ if they attempt to join the same revision more than once. Our use of a special error state is important to guarantee determinacy. Suppose two revisions try to join a third revision simultaneously (i.e. there is a race between two joins). Without the rule (join $\left.{ }_{\epsilon}\right)$ the different schedules may lead to different final states. However, with $\left(\right.$ join $\left._{\epsilon}\right)$, all executions are forced to eventually end up at $\epsilon$, maintaining determinacy.

To prepare for the proof, we now state and prove a local determinism lemma and a confluence theorem.

Lemma 1 (Local Determinism). If $s_{1} \approx s_{1}^{\prime}$ and $s_{1} \rightarrow_{r} s_{2}$ and $s_{1}^{\prime} \rightarrow_{r} s_{2}^{\prime}$, then $s_{2} \approx s_{2}^{\prime}$. 
Proof. First we observe that by construction, each evaluation context $\mathcal{E}$ contains at most one hole and that there is no choice in which redex to evaluate next. We can now do a case analysis on $\mathcal{E}[e]$ where $e$ is a redex. For a fixed revision $r$, such expression context is matched uniquely by at most one operational rule. Moreover, each rule is deterministic modulo $\alpha \beta$-equivalence. This is trivial for all operations except (new) and (fork) that create new locations and revisions respectively. Given a state $s(r \mapsto\langle\sigma, \tau, \mathcal{E}[$ ref $v]\rangle)$, rule $($ new $)$ can create different names for the new location, i.e. $s=s(r \mapsto\langle\sigma, \tau[l \mapsto$ $v], \mathcal{E}[l]\rangle)$ or $s^{\prime}=s\left(r \mapsto\left\langle\sigma, \tau\left[l^{\prime} \mapsto v\right], \mathcal{E}\left[l^{\prime}\right]\right\rangle\right)$. If $l=l^{\prime}$ this is equivalent directly. If $l \neq l^{\prime}$ we can apply $\alpha$-renaming with $\alpha=\left[l / l^{\prime}\right]$ where $s=\alpha\left(s^{\prime}\right)$ which holds since $l^{\prime} \notin s^{\prime}$ and $l \notin s$ due to the side condition on (new) (and by definition $s \approx s^{\prime}$ ). We prove equivalence similarly for (fork).

Lemma 2 (Strong Local Confluence). Let $s_{1}$ and $s_{1}^{\prime}$ be reachable states that satisfy $s_{1} \approx s_{1}^{\prime}$. Then, if $s_{1} \rightarrow_{r} s_{2}$ and $s_{1}^{\prime} \rightarrow_{r^{\prime}} s_{2}^{\prime}$, then there exist equivalent states $s_{3} \approx s_{3}^{\prime}$ such that both $s_{2} \rightarrow \stackrel{?}{r^{\prime}} s_{3}$ and $s_{2}^{\prime} \rightarrow \stackrel{?}{r} s_{3}^{\prime}$.

Proof. First we observe that when $r=r^{\prime}$, the lemma follows directly from the localdeterminism lemma. We continue the proof for the case $r \neq r^{\prime}$, and do a case distinction on the kind of the two operational steps appearing in the assumption of the theorem. We use the term local step to denote a step that is not (fork), (join), and (join $)_{\epsilon}$.

- (local) / (local). The rules affect independent parts of the state $s$ and thus commute. As before, we may need to use $\alpha$-renaming for the (new) case.

- (local) / (fork),(join). Same argument; note that the forked/joined revision can not be the same as the local one because of the side condition $r^{\prime} \notin s$ (for fork) or because the joinee can not take a step (for join).

- $\left(\right.$ join $\left._{\epsilon}\right) /$ any. The claim follows because if we could apply $\left(\right.$ join $\left._{\epsilon}\right)$ in some state but perform a different rule, then $\left(\right.$ join $\left._{\epsilon}\right)$ still applies.

- (fork) / (fork). In this case the side condition $r^{\prime} \notin s$ ensures that both forks will fork a unique revision. As shown in the proof of the previous lemma, we can safely apply $\beta$-renaming to show both end states are equivalent.

- (fork) / (join). Observe that the (join) cannot join on the revision that forks (since its expression is not a value). Also, the side condition $r^{\prime} \notin s$ ensures that a unique revision is forked that is different from $r$ and $r^{\prime}$ in the (join) rules.

- (join) / (join). The matched states are $s\left(r \mapsto\left\langle\sigma, \tau, \mathcal{E}\left[\right.\right.\right.$ rjoin $\left.\left.\left.r_{1}\right]\right\rangle\right)\left(r_{1} \mapsto\left\langle\sigma_{1}, \tau_{1}, v_{1}\right\rangle\right)$ and $s\left(r^{\prime} \mapsto\left\langle\sigma^{\prime}, \tau^{\prime}, \mathcal{E}^{\prime}\right.\right.$ [rjoin $\left.\left.\left.r_{2}\right]\right\rangle\right)\left(r_{2} \mapsto\left\langle\sigma_{2}, \tau_{2}, v_{2}\right\rangle\right)$. We have two possibilities. First, if $r_{1} \neq r_{2}$, both joins commute directly. Otherwise, $r_{1}=r_{2}$. In this case the joinee is shared. Thus, taking step $\rightarrow_{r}$ leads to a state where $s\left(r_{1} \mapsto \perp\right)$ and step $\rightarrow r^{\prime}$ must use $\left(\right.$ join $_{\epsilon}$ ) ending in state $\epsilon$, which is also the outcome for the opposite order.

Theorem 2 (Confluence). For any reachable states $s_{1} \approx s_{1}^{\prime}$, it holds that if $s_{1} \rightarrow^{*} s_{2}$ and $s_{1}^{\prime} \rightarrow^{*} s_{2}^{\prime}$, then there exist equivalent states $s_{3} \approx s_{3}^{\prime}$ such that both $s_{2} \rightarrow^{*} s_{3}$ and $s_{2}^{\prime} \rightarrow^{*} s_{3}^{\prime}$.

Proving confluence from strong local confluence is well-known and often illustrated using tiling of diagrams. It is useful for several applications (e.g. the lambda calculus or general term rewriting) but can also be understood more abstractly as a property of binary relations [18]. We include a quick proof sketch for reference. 
Proof. First, lift the step relation $\rightarrow$ to equivalence classes of states modulo $\approx$. Let $x, y, z, u$ range over equivalence classes, and consider the following three properties:

1. $\forall x y z: x \rightarrow y \wedge x \rightarrow z \Rightarrow \exists u: y \rightarrow^{?} u \wedge z \rightarrow^{?} u$

2. $\forall n: \forall x y z: x \rightarrow^{?} y \wedge x \rightarrow^{n} z \Rightarrow \exists u: y \rightarrow^{*} u \wedge z \rightarrow^{?} u$

3. $\forall n: \forall x y z: x \rightarrow^{n} y \wedge x \rightarrow^{*} z \Rightarrow \exists u: y \rightarrow^{*} u \wedge z \rightarrow^{*} u$

We can then show that (1) the first claim follows from strong local confluence, (2) the second claim follows from the first by induction over $n$, (3) the third claim follows from the second by induction over $n$, and (4) the theorem follows from the third claim.

We now conclude with the proof of theorem 1. Given a program expression $e$ and two maximal executions $s_{0} \rightarrow^{*} s$ and $s_{0}^{\prime} \rightarrow^{*} s^{\prime}$ for $e$, we know $s_{0} \approx s_{0}^{\prime}$ (by the way we defined initial states for $e$ ), so by the confluence theorem there exist $s_{1} \approx s_{1}^{\prime}$ such that $s \rightarrow^{*} s_{1}$ and $s^{\prime} \rightarrow^{*} s_{1}^{\prime}$. But since $s$ and $s^{\prime}$ are maximal it must be the case that $s=s_{1}$ and $s^{\prime}=s_{1}^{\prime}$ and thus $s \approx s^{\prime}$ as claimed.

\section{State Merging}

The basic calculus introduced in the previous section provides little flexibility as to how write-write conflicts should be resolved. We now show how to modify the calculus so that it can support custom merge functions (Section 4.1), how it can be understood as an extension of snapshot isolation (Section 4.3), and how we can provide stronger consistency guarantees for abstract data types using sequential merge functions (Section 4.4).

$$
\begin{aligned}
& \text { (join-merge) } s\left(r \mapsto\left\langle\sigma, \tau, \mathcal{E}\left[\text { rjoin } r^{\prime}\right]\right\rangle\right)\left(r^{\prime} \mapsto\left\langle\sigma^{\prime}, \tau^{\prime}, v\right\rangle\right) \quad \rightarrow_{r} \\
& s\left[r \mapsto\left\langle\sigma, \operatorname{merge}\left(\tau, \tau^{\prime}, \sigma^{\prime}\right), \mathcal{E}[\text { unit }]\right\rangle\right]\left[r^{\prime} \mapsto \perp\right] \\
& \text { where } \quad \operatorname{merge}\left(\tau, \tau^{\prime}, \sigma^{\prime}\right)(l)= \begin{cases}\tau(l) & \text { if } \tau^{\prime}(l)=\perp \\
\tau^{\prime}(l) & \text { if } \sigma^{\prime}(l)=\tau(l) \\
\text { merge }_{l}\left(\tau(l), \tau^{\prime}(l), \sigma^{\prime}(l)\right) & \text { otherwise }\end{cases}
\end{aligned}
$$

Fig. 5. Extending the revision calculus with merge functions

\subsection{Merge Functions}

Figure 5 extends the basic calculus with flexible merge functions. There is just one change to the basic calculus where we replace the (join) rule with the (join-merge) rule. Instead of composing the new state as $\tau:: \tau^{\prime}$ we call a custom merge $\left(\tau, \tau^{\prime}, \sigma^{\prime}\right)$ function that merges the states. If there is no (write-write) conflict at a particular location, this function behaves just like our earlier composition. In case of conflict, the value at a location $l$ after a join is determined by a location specific function merge $\mathrm{I}_{l}: \mathrm{Val} \times \mathrm{Val} \times$ $V a l \rightarrow V a l$ which is defined separately for each location $l$.

Note that the choice of merge function does not influence determinacy. The determinacy proof remains intact regardless of what merge function is chosen (as long as it is a function of its three inputs). In particular, we need not restrict our attention to commutative or associative functions only. 


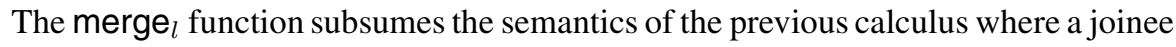
takes precedence since we can define the default merge function as:

$$
\operatorname{merge}_{l}\left(v, v^{\prime}, v_{0}\right)=v^{\prime} \quad \text { (joinee wins) }
$$

Similarly, we can implement the dual strategy where updates to a specific location are ignored if there is a write-write conflict:

$$
\operatorname{merge}_{l}\left(v, v^{\prime}, v_{0}\right)=v \quad \text { (joiner wins) }
$$

Note that sometimes, we may wish to define merge functions involving more than a single variable. In our calculus we can do so by using composite types to group several variables into a single location and merge them collectively.

\subsection{Commutative Merges}

We call a merge function commutative if $\operatorname{merge}_{l}\left(v, v^{\prime}, v_{0}\right)=\operatorname{merge}_{l}\left(v^{\prime}, v, v_{0}\right)$. Clearly, the default merge function is not commutative, but many others are. For example, a reasonable merge function for sets could be:

$$
\operatorname{merge}_{l}\left(s, s^{\prime}, s_{0}\right)=s \cup s^{\prime}
$$

which is commutative. This is not the only reasonable merge function though. Consider the following venn diagram that shows how the sets $s, s^{\prime}$, and $s_{0}$ may interact:

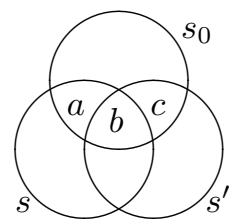

When taking the union of $s$ and $s^{\prime}$, we always include the regions $a, b$, and $c$. One can argue however that to end up with $s^{\prime}$ from $s_{0}$, the elements in $a$ were explicitly removed (and similarly for $s$ with region $c$ ). Another reasonable merge function may respect such removals and remove region $a$ and $c$ from the final result. We can specify this as:

$$
\operatorname{merge}_{l}\left(s, s^{\prime}, s_{0}\right)=\left(s-s_{0}\right) \cup\left(s^{\prime}-s_{0}\right) \cup\left(s \cap s^{\prime}\right)
$$

which is also commutative. Note that when all operations on the set are additive, both of these merge functions produce the same result since $s_{0} \subseteq\left(s \cap s^{\prime}\right)$ in that case.

Ultimately, this discussion simply illustrates that the choice of a merge function should be informed by what operations are performed (additions only, removals only, both, etc.). We discuss this idea more formally in Section 4.4 , where we show that by restricting the operations on an abstract data type, we can find merge functions can provide particularly strong guarantees. 


$$
\begin{array}{llr}
\text { (join-ok) } & s\left(r \mapsto\left\langle\sigma, \tau, \mathcal{E}\left[\text { rjoin } r^{\prime}\right]\right\rangle\right)\left(r^{\prime} \mapsto\left\langle\sigma^{\prime}, \tau^{\prime}, v\right\rangle\right) & \rightarrow_{r} \\
& s\left[r \mapsto\left\langle\sigma, \operatorname{merge}\left(\tau, \tau^{\prime}, \sigma^{\prime}\right), \mathcal{E}[\text { true }]\right\rangle\right]\left[r^{\prime} \mapsto \perp\right] & \text { if } \neg \text { fail }\left(\tau, \tau^{\prime}, \sigma^{\prime}\right) \\
\text { (join-fail }) & s\left(r \mapsto\left\langle\sigma, \tau, \mathcal{E}\left[\text { rjoin } r^{\prime}\right]\right\rangle\right)\left(r^{\prime} \mapsto\left\langle\sigma^{\prime}, \tau^{\prime}, v\right\rangle\right) & \rightarrow_{r} \\
& s[r \mapsto\langle\sigma, \tau, \mathcal{E}[\text { false }]\rangle]\left[r^{\prime} \mapsto \perp\right] & \text { if fail }\left(\tau, \tau^{\prime}, \sigma^{\prime}\right) \\
\text { where } & \text { fail }\left(\tau, \tau^{\prime}, \sigma^{\prime}\right)=\text { undef } \in \operatorname{rng}\left(\operatorname{merge}\left(\tau, \tau^{\prime}, \sigma^{\prime}\right)\right) &
\end{array}
$$

Fig. 6. Extending the merge calculus with failing joins

\subsection{Snapshot Isolation}

We now explain how to view our system as a generalization of snapshot isolation [4], a concurrency control algorithm that is widely used in the database community, and has for example been implemented by Oracle and Microsoft SQL Server (with minor variations). We use the definition given by Fekete et al. [11].

We claim that our revision calculus is a generalization of snapshot isolation, augmented by (1) the ability to gracefully resolve write-write conflict when a suitable merge function exists for a particular location, and (2) support nontrivial nesting (Fig. 3) while maintaining a simple and precise semantics. To see why this is the case, we perform the reverse process: we (1) introduce the ability to fail on write-write conflicts, and (2) remove nesting from revisions.

Removing nesting is straightforward (for example, we can disallow forks by all revisions but the main revision). As for failing on conflicts, we proceed as follows. To mirror how transactions fail (and discard state), we introduce the notion of a failing join as follows.

- We change the merge calculus slightly, by redefining the local merge functions so that they can return a special value indicating that there is an unresolvable conflict:

$$
\text { merge }_{l}: \text { Val } \times \text { Val } \times \text { Val } \rightarrow(\text { Val } \cup\{\text { undef }\})
$$

- We extend the merge calculus by replacing (join-merge) with two new rules. The (join-ok) rule is equivalent to the previous (join-merge) rule but can only be applied now if all of the location specific merge functions are defined. The rule (join-fail) applies if at least one of the merges failed and simply ignores all updates in the joinee. Both rules now return a boolean to the joiner, where true indicates that the join was successful, and false indicates that it was not.

Consider now the definition of snapshot isolation: A transaction A executing under snapshot isolation operates on a snapshot of the database taken at the start of the transaction. When the transaction concludes, it will successfully commit only if the values updated by the transaction A were not updated by any other transaction B that committed after transaction A started.

We can succinctly describe this behaviour in our calculus by letting every merge ${ }_{l}$ function fail:

$$
\operatorname{merge}_{l}\left(v, v^{\prime}, v_{0}\right)=\text { undef } \quad \text { (snapshot isolation) }
$$


When discussing snapshot isolation there is sometimes confusion whether a transaction should abort if there was a concurrent silent write in the main branch where the original value has been left unchanged. In our formal calculus there is no such confusion: due to the second case of the merge function (Fig. 5), concurrent silent writes on the main branch will not cause a transaction to fail. Note that we can still model the behaviour where silent writes cause a transaction to fail by assigning sequence numbers to each value (ensuring that $\sigma^{\prime}(l) \neq \tau(l)$ on silent writes). Dually, we can ignore silent writes on the child branch by modifying the merge function:

$$
\operatorname{merge}_{l}\left(v, v^{\prime}, v_{0}\right)=\left(\left(v^{\prime}=v_{0}\right) ? v^{\prime}: \text { undef }\right) \quad \text { (ignore silent wr) }
$$

\subsection{Abstract Data Types and Sequential Merges}

As Theorem 1 shows, our calculus is always determinate, but we have seen in the introduction that it is not always serializable (Fig. 2). However, we can sometimes guarantee equivalence to a sequential execution by raising the abstraction level of operations on data, and constructing merge functions that are tailored to the operations that are performed.

For example, consider a program location $x$ that is initially zero and for which we define the merge function $\operatorname{merge}_{x}\left(v, v^{\prime}, v_{0}\right)=v+v^{\prime}-v_{0}$. Furthermore, assume that a program performs only one type of operation on $x$, namely add $(i)$, which adds an integer $i$ to it. Then the final value of $x$ is always consistent with a serial execution of all the add operations that occurred in the program. We now explain this idea more formally.

Abstract Data Types. We define an abstract data type to be a tuple $(V, o, O p, o p)$ where $V$ is a set of values, $o \in V$ is an initial value, $O p$ is a set of operations, and $o p: O p \times V \rightarrow V$ is a partial function. In our formalization, the set $O p$ includes argument and return values of operations, and $o p$ is partial because not all operations apply in all states.

Example 1. We can define an integer register (i.e. a memory location holding an integer that can be read and written) as IntReg $=(\mathbb{Z}, 0, O p, o p)$ where

$$
\begin{aligned}
o p & =\{\operatorname{get}(v) \mid v \in \mathbb{Z}\} \cup\{\operatorname{set}(v) \mid v \in \mathbb{Z}\} \\
o p(v, o) & =\left\{\begin{array}{l}
w \text { if } o=\operatorname{set}(w) \\
v \text { if } o=\operatorname{get}(v) \\
\perp \text { if } o=\operatorname{get}\left(v^{\prime}\right) \text { and } v \neq v^{\prime}
\end{array}\right.
\end{aligned}
$$

Sequential Merge Functions. Sometimes we can find merge functions that can simulate a deterministic, linear interleaving of the operations. We call such merge functions sequential. This concept is quite useful in practice since the programmer can design the application specifically to enable sequential merge functions, by restricting what type of operations may happen in concurrent revisions. For example, if an application performs aggregation of results, sequential merges usually exist. 
To study the effect of entire sequences of operations, we introduce the following concise notations. We consider operation sequences as words in $O p^{*}$, and write $u(v)$ (where $u \in O p^{*}$ and $v \in V a l$ ) for the combined effect of all the operations in the sequence $u$ (left to right) applied to the value $v$, which may be undefined. For example, this means that for operation sequences $u, w \in O p^{*}$ and a value $v$, we have $u w(v)=$ $w(u(v))$ if $u(v) \neq \perp$ and $w(u(v)) \neq \perp$. We now define sequential merge functions as:

Definition 1. Let $\mathcal{A}=(V, o, O p, o p)$ be an abstract data type. We say a merge function $\mathrm{m}: V \times V \times V \rightarrow V$ is sequential for $\mathcal{A}$ iffor all operation sequences $u, w_{1}, w_{2} \in O p^{*}$ such that $u(o) \neq \perp, u w_{1}(o) \neq \perp$ and $u w_{2}(o) \neq \perp$, both of the following are true:

1. $u w_{1} w_{2}(o) \neq \perp$

2. $\mathrm{m}\left(u w_{1}(o), u w_{2}(o), u(o)\right)=u w_{1} w_{2}(o)$

The advantage of a sequential merge function is that it guarantees the appearance that all operations were executed sequentially, with the operations of the joined revision happening at the time of the join.

Note that condition 1 of Def. 1 does not depend on the actual merge function, but is a property of the abstract data type. This property may not be satisfiable, thus sequential merge functions do not exist for all abstract data types. For example, the abstract data type IntReg defined in Example 1 does not permit sequential merging because it can be the case that $w_{1}=\operatorname{set}(1), w_{2}=\operatorname{get}(0)$ in which case always $u w_{1} w_{2}(0)=\perp$.

Abelian Data Types. Particularly simple to merge are certain abstract data types with commutative operations that we call abelian. More formally, call an abstract data type $(V, o, O p, o p)$ abelian if there exists a binary operation + on $V$, and a function $\delta$ : $O p \rightarrow V$ such that (1) $(V,+)$ is an abelian group with neutral element $o$, and (2) for all $a \in O p$ we have $a(v)=v+\delta(a)$.

We conclude this section with a lemma that shows how to construct sequential merge functions for abelian data types.

Lemma 3. For an abelian data type $(V, o, O p, o p)$ with operation + , the merge function : $\mathrm{m}\left(v_{1}, v_{2}, v\right)=v_{1}+v_{2}-v$ is sequential.

Proof. Let $w_{1}=\sum_{i=1}^{n} a_{i}$ and $w_{2}=\sum_{i=1}^{m} b_{i}$ and $v=u(o)$. Then claim 2 is satisfied: $\mathrm{m}\left(u w_{1}(o), u w_{2}(o), u(o)\right)=\mathrm{m}\left(w_{1}(u(o)), w_{2}(u(o)), u(o)\right)=\mathrm{m}\left(v+\sum_{i=1}^{n} a_{i}, v+\right.$ $\left.\sum_{i=1}^{m} b_{i}, v\right)=v+\sum_{i=1}^{n} a_{i}+v+\sum_{i=1}^{m} b_{i}-v=v+\sum_{i=1}^{n} a_{i}+\sum_{i=1}^{m} b_{i}=$ $w_{1} w_{2}(u(o))=u w_{1} w_{2}(o)$. This implies also that claim 1 is satisfied.

\section{Revision Diagrams}

In this section we describe and formally define revision diagrams, a special kind of graph that visually represent the dataflow of computations of our calculus. Revision diagrams are an essential tool to understand how to program with revisions, somewhat analogous to the role of stream diagrams for stream programming. Revision diagrams are not equivalent to other graph classes commonly used for representing concurrent 


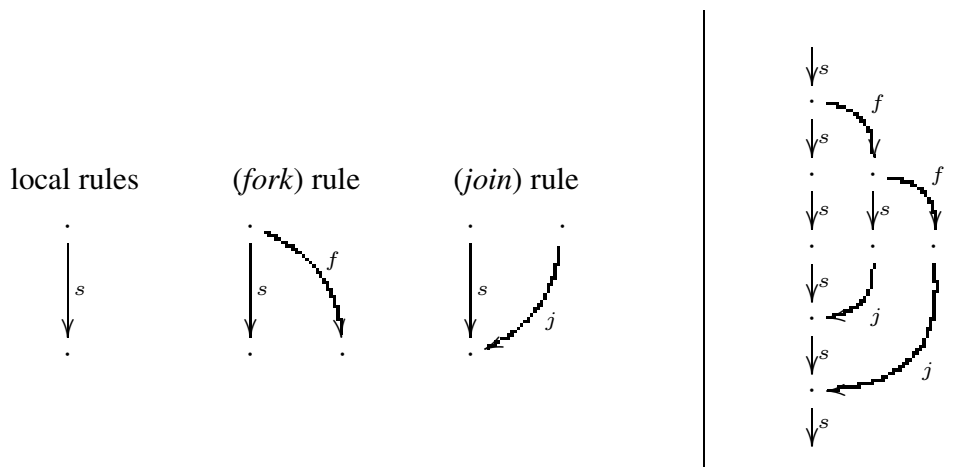

Fig. 7. (a) Left: the diagrams illustrate how revision diagrams are constructed incrementally by transition rules adding vertices. (b) Right: a typical example of a revision diagram.

or parallel qcomputations, such as directed acyclic graphs (DAGs), or series-parallel graphs (SP-graphs). The goal of this section is to formally define revision diagrams and to shed more light on their general structure.

Intuitively, revision diagrams represent executions, with vertices being states and edges being transitions. Technically, revision diagrams are labeled graphs:

Definition 2. $A$ fsj-graph $G$ is a tuple $G=(V, E)$ where $V$ is a set of vertices and $E \subset V \times\{f, s, j\} \times V$ is a set of labeled edges.

Graph Notations. We use the usual terminology for graphs, but emphasize a relational view of edges. For a fixed graph $G=(V, E)$, we define a binary relation $\stackrel{f}{\rightarrow}$ on vertices such that $(u \stackrel{f}{\rightarrow} v) \stackrel{\text { def }}{\Leftrightarrow}((u, f, v) \in E)$, and similarly for $\stackrel{j}{\rightarrow}$ and $\stackrel{s}{\rightarrow}$. We also define the relation $\rightarrow \stackrel{\text { def }}{=}(\stackrel{f}{\rightarrow} \cup \stackrel{j}{\rightarrow} \cup \stackrel{s}{\rightarrow})$.

To define the revision diagram for a given execution, we extend the original operational semantics so that a fsj-graph is constructed alongside the executing program. More formally, we extend the original transition relation $\rightarrow$ to an extended transition relation $\rightarrow_{d}$ (where $d$ is just a label for easier distinction) on states $(s,(V, E), \rho, \gamma)$ where: $s \in$ GlobalState is a global state as defined previously, $(V, E)$ is a fsj-graph, $\rho: V \rightarrow$ Rid maps vertices to revision they belong to, and $\gamma: \operatorname{Rid} \rightarrow V$ is a partial function that maps a revision to the last (current) vertex of that revision.

The graph is constructed incrementally by adding new vertices, and edges from the existing graph to the new vertices, as illustrated in Fig.7 (a). The precise transition rules for $\rightarrow_{d}$ are defined in Fig. 8 Intutively, the constructed graphs represents excecutions in the following sense (for an example, see Fig. 7 (b)):

- Each vertex $v \in V$ belongs to a particular revision $\rho(v)$ and represents the local state of that revision at a certain point of time.

- The set of all vertices belonging to the same revisions is totally ordered by $\stackrel{s}{\rightarrow}$, the successor relation, which describes how the local state of that revision evolves over time.

- Each edge in $\stackrel{f}{\rightarrow}$ represents the forking of a new revision. Its destination vertex is the first vertex of the new revision. 
- Each edge in $\stackrel{j}{\rightarrow}$ represents the joining of a revision. Its source vertex is that last vertex of the revision being joined.

We define the initial states to be $\left(s_{0}, G_{0}, \rho_{0}, \gamma_{0}\right)$ where $s_{0}=(r,(\epsilon, \epsilon, e))$ is an initial global state (that is, $r$ is a revision identifier and $e$ is an expression not containing any revision identifiers), $G_{0}=(\{v\}, \emptyset)$ is a singleton graph, and $\rho_{0}=\{v \mapsto r\}$, $\gamma_{0}=\{r \mapsto v\}$. We say a state $(s, G, \rho, \gamma)$ is reachable if there exists an initial state from which it can be reached by zero or more $\rightarrow{ }_{d}$-transitions.

Definition 3. A revision diagram is an $f$ sj-graph $G$ that is part of some reachable state $(s, G, \rho, \gamma)$.

It is easy to see (comparing the definitions of $\rightarrow$ and $\rightarrow_{d}$ ) that for any execution $s_{0} \rightarrow^{*} s_{n}$ such that $s_{n} \neq \epsilon$, we can find a corresponding extended execution, and vice versa. Note that this may entail the renaming of revision identifiers in the $\rightarrow$-execution, because the latter does allow the reuse of revision identifiers after the revision has been joined while $\rightarrow_{d}$ does not. Our main theorem can now be stated as:

$$
\begin{aligned}
& \text { (apply) } \quad(s(r \mapsto\langle\sigma, \tau, \mathcal{E}[(\lambda x . e) v]\rangle),(V, E), \rho, \gamma) \quad \rightarrow_{d} \quad(\text { if } v \notin V) \\
& (s[r \mapsto\langle\sigma, \tau, \mathcal{E}[[v / x] e]\rangle],(V \cup v, E \cup \gamma(r) \stackrel{s}{\rightarrow} v), \rho[v \mapsto r], \gamma[r \mapsto v]) \\
& \text { (if-true) }\left(s\left(r \mapsto\left\langle\sigma, \tau, \mathcal{E}\left[\left(\text { true } ? e_{1}: e_{2}\right)\right]\right\rangle\right),(V, E), \rho, \gamma\right) \quad \rightarrow_{d} \quad(\text { if } v \notin V) \\
& \left(s\left[r \mapsto\left\langle\sigma, \tau, \mathcal{E}\left[e_{1}\right]\right\rangle\right],(V \cup v, E \cup \gamma(r) \stackrel{s}{\rightarrow} v), \rho[v \mapsto r], \gamma[r \mapsto v]\right) \\
& \text { (if-false) }\left(s\left(r \mapsto\left\langle\sigma, \tau, \mathcal{E}\left[\left(\text { false } ? e_{1}: e_{2}\right)\right]\right\rangle\right),(V, E), \rho, \gamma\right) \quad \rightarrow_{d} \quad(\text { if } v \notin V) \\
& \left(s\left[r \mapsto\left\langle\sigma, \tau, \mathcal{E}\left[e_{2}\right]\right\rangle\right],(V \cup v, E \cup \gamma(r) \stackrel{s}{\rightarrow} v), \rho[v \mapsto r], \gamma[r \mapsto v]\right) \\
& \text { (new) } \quad(s(r \mapsto\langle\sigma, \tau, \mathcal{E}[\operatorname{ref} v]\rangle),(V, E), \rho, \gamma) \quad \rightarrow_{d} \quad(\text { if } l \notin s \text { and } v \notin V) \\
& (s[r \mapsto\langle\sigma, \tau[l \mapsto v], \mathcal{E}[l]\rangle],(V \cup v, E \cup \gamma(r) \stackrel{s}{\rightarrow} v), \rho[v \mapsto r], \gamma[r \mapsto v]) \\
& \text { (get) } \quad(s(r \mapsto\langle\sigma, \tau, \mathcal{E}[! l]\rangle),(V, E), \rho, \gamma) \quad \rightarrow_{d} \quad(\text { if } l \in \operatorname{dom}(\sigma:: \tau) \text { and } v \notin V) \\
& (s[r \mapsto\langle\sigma, \tau, \mathcal{E}[(\sigma:: \tau)(l)]\rangle](V \cup v, E \cup \gamma(r) \stackrel{s}{\rightarrow} v), \rho[v \mapsto r], \gamma[r \mapsto v]) \\
& (s e t) \quad(s(r \mapsto\langle\sigma, \tau, \mathcal{E}[l:=v]\rangle),(V, E), \rho, \gamma) \quad \rightarrow_{d} \quad(\text { if } l \in \operatorname{dom}(\sigma:: \tau) \text { and } v \notin V) \\
& (s[r \mapsto\langle\sigma, \tau[l \mapsto v], \mathcal{E}[\text { unit }]\rangle],(V \cup v, E \cup \gamma(r) \stackrel{s}{\rightarrow} v), \rho[v \mapsto r], \gamma[r \mapsto v]) \\
& \text { (fork) } \left.\quad(s(r \mapsto\langle\sigma, \tau, \mathcal{E} \text { [rfork } e]\rangle)(V, E), \rho, \gamma) \rightarrow_{d} \text { (if } r^{\prime} \notin s, v, w \notin V \text { and } r^{\prime} \notin \mathrm{rng}(\rho)\right) \\
& \left(s\left[r \mapsto\left\langle\sigma, \tau, \mathcal{E}\left[r^{\prime}\right]\right\rangle\right]\left[r^{\prime} \mapsto\langle\sigma:: \tau, \epsilon, e\rangle\right],\right. \\
& \left.(V \cup\{v, w\}, E \cup\{\gamma(r) \stackrel{s}{\rightarrow} v, \gamma(r) \stackrel{f}{\rightarrow} w\}), \rho[v \mapsto r]\left[w \mapsto r^{\prime}\right], \gamma[r \mapsto v]\left[r^{\prime} \mapsto w\right]\right) \\
& \text { (join) } \quad\left(s\left(r \mapsto\left\langle\sigma, \tau, \mathcal{E}\left[\text { rjoin } r^{\prime}\right]\right\rangle\right)\left(r^{\prime} \mapsto\left\langle\sigma^{\prime}, \tau^{\prime}, v\right\rangle\right)(V, E), \rho, \gamma\right) \quad \rightarrow_{d} \quad(\text { if } v \notin V) \\
& \left(s\left[r \mapsto\left\langle\sigma, \tau:: \tau^{\prime}, \mathcal{E}[\text { unit }]\right\rangle\right]\left[r^{\prime} \mapsto \perp\right],\right. \\
& \left.\left(V \cup v, E \cup\left\{\gamma(r) \stackrel{s}{\rightarrow} v, \gamma\left(r^{\prime}\right) \stackrel{j}{\rightarrow} v\right\}\right), \rho[v \mapsto r], \gamma[r \mapsto v]\left[r^{\prime} \mapsto \perp\right]\right)
\end{aligned}
$$

Fig. 8. Operational rules for $\rightarrow_{d}$. The rules match the ones in Fig. 4] except for the highlighted parts, and for the omission of $\left(\right.$ join $\left._{\epsilon}\right)$.

Theorem 3. Let $G=(V, E)$ be a revision diagram. Then $G$ is a semilattice, i.e. for any two vertices $x, y \in V$, there exists a greatest common ancestor. 
While plausible, this result is not obvious, as it hinges on where exactly revisions can be joined from: If we could join revisions from anywhere (e.g. if we used sequential consistency without isolating revisions), the graphs would be general DAGs which are not always semilattices (e.g. see Fig. 3, on the right). On the other hand, if our revisions were simply nested like trees (as enforced in Cilk, for example), the resulting graphs would be SP-graphs which can be easily proved to always be semilattices. For revision diagrams, however, the proof of the semilattice property is somewhat subtle as it depends on the fact that revisions can be joined only from locations that are downstream from the fork (since revision handles must flow along lines). For a detailed proof of this theorem as well as other useful or interesting properties of revision diagrams see the companion Tech Report [9].

\section{Conclusion and Future Work}

We have presented a novel programming model based on concurrent revisions. First, we presented a concise calculus that shows how revisions can maintain determinacy despite nondeterministic scheduling. Then we provided a discussion of how state merging can be tailored to the needs of the application. Finally, we formalized revision diagrams, the fundamental tool to visualize nonlinear histories of state, and showed how they differ from other task graphs. In future work, we may further investigate state merging and serialization guarantees. We are also interested in enhancing the calculus with reactive inputs and outputs and extending the determinacy guarantee to such applications.

Acknowledgments. We thank Tim Harris, Tom Ball and Manuel Fähndrich for their helpful feedback.

\section{References}

1. Aditya, S., Arvind, Augustsson, L., Maessen, J.-W., Nikhil, R.: Semantics of pH: A Parallel Dialect of Haskell. In: Hudak, P. (ed.) Proc. Haskell Workshop, La Jolla, CA, USA, pp. 35-49 (June 1995)

2. Allen, E., Chase, D., Flood, C., Luchangco, V., Maessen, J.-W., Ryu, S., Steele Jr., G.: Project fortress: A multicore language for multicore processors. In: Linux Mag. (September 2007)

3. Baldassin, A., Burckhardt, S.: Lightweight software transactions for games. In: Workshop on Hot Topics in Parallelism, HotPar (2009)

4. Berenson, H., Bernstein, P., Gray, J., Melton, J., O'Neil, E., O’Neil, P.: A critique of ANSI SQL isolation levels. In: Proceedings of SIGMOD, pp. 1-10 (1995)

5. Berger, E., Yang, T., Liu, T., Novark, G.: Grace: Safe multithreaded programming for C/C++. In: OOPSLA (2009)

6. Blelloch, G., Chatterjee, S., Hardwick, J., Sipelstein, J., Zagha, M.: Impl. of a portable nested data-parallel language. Journal of Par. and Dist. Comp. 21(1), 4-14 (1994)

7. Bocchino, R., Adve, V., Dig, D., Adve, S., et al.: A type and effect system for deterministic parallel java. In: OOPSLA (2009)

8. Burckhardt, S., Baldassin, A., Leijen, D.: Concurrent programming with revisions and isolation types. In: OOPSLA (October 2010)

9. Burckhardt, S., Leijen, D.: Semantics of concurrent revisions. Technical Report MSR-TR2010-94, Microsoft Research (2010) 
10. Denning, P., Dennis, J.: The resurgence of parallelism. Commun. ACM 53(6) (2010)

11. Fekete, A., Liarokapis, D., O’Neil, E., O’Neil, P., Shasha, D.: Making snapshot isolation serializable. ACM Trans. Database Syst. 30(2), 492-528 (2005)

12. Flanagan, C., Felleisen, M.: The semantics of future and its use in program optimization, Rice University, pp. 209-220 (1995)

13. Frigo, M., Halpern, P., Leiserson, C.E., Lewin-Berlin, S.: Reducers and other cilk++ hyperobjects. In: Sym. on Par. Algorithms and Architectures, SPAA, pp. 79-90 (2009)

14. Frigo, M., Leiserson, C., Randall, K.: The implementation of the Cilk-5 multithreaded language. In: Programming Language Design and Impl., PLDI, pp. 212-223 (1998)

15. Harris, T., Cristal, A., Unsal, O., Ayguadé, E., Gagliardi, F., Smith, B., Valero, M.: Transactional memory: An overview. IEEE Micro 27(3), 8-29 (2007)

16. Herlihy, M., Koskinen, E.: Transactional boosting: a methodology for highly-concurrent transactional objects. In: Principles and Practice of Parallel Programming, PPoPP, pp. 207216 (2008)

17. Herlihy, M., Wing, J.: Linearizability: a correctness condition for concurrent objects. ACM Trans. Program. Lang. Syst. 12(3), 463-492 (1990)

18. Huet, G.: Confluent reductions: Abstract properties and applications in term rewriting systems. J. ACM 27(4) (October 1980)

19. Koskinen, E., Parkinson, M., Herlihy, M.: Coarse-grained transactions. In: Principles of Programming Languages, POPL, pp. 19-30 (2010)

20. Kulkarni, M., Pingali, K., Walter, B., Ramanarayanan, G., Bala, K., Chew, L.: Optimistic parallelism requires abstractions. In: PLDI (2007)

21. Lamport, L.: How to make a multiprocessor computer that correctly executes multiprocess programs. IEEE Trans. Comp. C-28(9), 690-691 (1979)

22. Larus, J., Rajwar, R.: Transactional Memory. Morgan \& Claypool (2007)

23. Lee, J., Palsberg, J.: Featherweight x10: a core calculus for async-finish parallelism. In: Principles and Practice of Parallel Programming, PPoPP 2010 (2010)

24. Martin, A., Birrell, A., Harris, T., Isard, M.: Semantics of transactional memory and automatic mutual exclusion. In: Principles of Prog. Lang. POPL, pp. 63-74 (2008)

25. Moreau, L.: The semantics of scheme with future. In: ACM SIGPLAN International Conference on Functional Programming, ICFP 1996, pp. 146-156 (1996)

26. Bernstein, P.A., Goodman, N.: Multiversion concurrency control-theory and algorithms. ACM Trans. Database Syst. 8(4), 465-483 (1983)

27. Bernstein, P.A., Hadzilacos, V., Goodman, N.: Concurrency Control and Recovery in Database Systems. Addison-Wesley, Reading (1987)

28. Pratikakis, P., Spacco, J., Hicks, M.: Transparent proxies for java futures. SIGPLAN Not. 39(10), 206-223 (2004)

29. Randall, K.: Cilk: Efficient Multithreaded Computing. PhD thesis, Dept. of Electrical Engineering and Computer Science. MIT, Cambridge (1998)

30. Riegel, T., Fetzer, C., Felber, P.: Snapshot isolation for software transactional memory. In: Workshop on Transactional Computing, TRANSACT (2006)

31. Steele, G.: Parallel programming and parallel abstractions in fortress. In: Hagiya, M. (ed.) FLOPS 2006. LNCS, vol. 3945, pp. 1-1. Springer, Heidelberg (2006)

32. Welc, A., Jagannathan, S., Hosking, A.: Safe futures for java. In: OOPSLA, pp. 439-453 (2005)

33. Welc, A., Saha, B., Adl-Tabatabai, A.-R.: Irrevocable transactions and their applications. In: Symposium on Parallel Algorithms and Architectures, SPAA, pp. 285-296 (2008) 